\title{
Pertinencia de un Programa Docente ante los retos de la Industria 4.0
}

\section{Relevance of a Teaching Program to the challenges of Industry 4.0}

\author{
José Daniel Corona-Flores \\ Xochitl Ruelas-Chacón \\ Oscar Rebolloso-Padilla \\ Laura Fuentes-Lara \\ Antonio Aguilera-Carbó \\ Universidad Autónoma Agraria Antonio Narro, Saltillo, México
}

\section{Resumen}

Objetivo: identificar el grado de pertinencia del Programa Docente de Ingeniero en Ciencia y Tecnología de Alimentos (PDICTA) para enfrentar los retos que demanda la Cuarta Revolución Industrial o Industria 4.0. Método: para la recolección de los datos se aplicó una encuesta como instrumento de medición, utilizando la escala de Likert de cinco opciones de respuesta a 10 ítems para una muestra representativa de 12 profesores y 14 egresados del programa. Para el análisis de los datos de la encuesta, se aplicó la estadística descriptiva. Resultados: los hallazgos fueron los siguientes: en 4 de los items, los profesores y egresados manifestaron estar de acuerdo, mientras que en los 6 items restantes, los resultados mostraron diferentes respuestas que estuvieron en niveles que variaron desde estar en desacuerdo, neutral y de acuerdo. Discusión y Conclusiones: tanto los profesores como los egresados, estuvieron de acuerdo en que los primeros apoyaron a los segundos en el desarrollo de cuatro de las diez habilidades o competencias que demanda la Industria 4.0, lo que indica que el PDICTA está en un proceso de cambio para incursionar en el mercado laboral que demanda la Industria 4.0.

Palabras clave: cuarta revolución industrial, educación 4.0, industria 4.0.

\section{Abstract}

Objective: to identify the level of relevance of the Food Science and Technology Engineering Teaching Program (PDICTA, for its Spanish acronym) to face the challenges demanded by the Fourth Industrial Revolution or Industry 4.0. Method: a survey was conducted as measurement instrument for data gathering, using a 5point Likert scale to answer 10 items for a representative sampling of 12 teachers and 14 program graduates. A descriptive statistics was applied for the analysis of the survey data. Results: the findings were the following: in 4 of the items, teachers and graduates stated "agree," while on the 6 remaining items, the answers varied between "disagree," "neutral," and "agree". Discussion and conclusion: both teachers and graduates agreed that the former supported the latter in the development of four out of the ten skills or abilities demanded by the Industry 4.0, which indicates that the PDICTA is in a process of change to make an incursion in the labor market demanded by Industry 4.0.

Keywords: education 4.0, industry 4.0, fourth industrial revolution.
Open Access:

E-ISSN: $2665-2420$ ISSN: $0124-2121$

ARTÍCULO RESULTADO DE INVESTIGACIÓN Copyright $\odot 2020$ By Educación y Humanismo Dhayana Fernández Matos Universidad Simón Bolívar

Recibido: 17-01-2020 Aceptado: 13-05-2020 En línea desde: 02-07-2020

Cómo citar este artículo (APA): Corona-Flores, J., Ruelas-Chacón, X., Rebolloso-Padilla, O., Fuentes-Lara, L., \& Aguilera-Carbó, Á. 


\section{Introducción}

La plataforma Industria 4.0 (I.4.0) es un concepto promovido principalmente por el Cluster Institute en el World Economic Forum Report (2016), y por actores relacionados como Klaus Schwab y el Grupo de Trabajo de Industria 4.0 (grupo formado por representantes de la empresa alemana Bosch y la Academia Alemana de Ciencias e Ingeniería), por lo que la mayor parte de los que escriben y promueven esta idea provienen principalmente de Alemania o están relacionados con la mencionada propuesta económica internacional.

Aunque esta plataforma se utiliza como sinónimo de manufactura altamente computarizada y automatizada, en realidad el término abarca una definición más amplia. La literatura relacionada es relativamente reciente, por lo que no existe una definición consensuada por todas las instituciones y actores principales. Hermann y Otto (2015) realizaron una revisión de la literatura en el tema y proponen una definición que engloba a varios autores y que se resume por seis palabras clave: interoperabilidad, virtualización, descentralización, capacidad en tiempo real, orientación a servicios y modularidad.

En este sentido, Shwab (2016) proporciona un ejemplo que ayuda a comprender cómo las revoluciones industriales cambiaron a lo largo del tiempo. Durante la primera Revolución Industrial (RI), se usaron agua y vapor para mecanizar la producción. Durante la segunda RI, la energía eléctrica se utilizó para crear producción en masa. Durante la tercera RI, la electrónica y las tecnologías de la información se usaron para automatizar la producción. La cuarta RI está más allá de una mejora de la tercera RI, en el que el avance de las nuevas tecnologías atenúa o desvanece las líneas entre los mundos físico, digital y biológico. Las nuevas tecnologías evolucionan a un ritmo exponencial y no existe un precedente histórico que marque el comienzo de la evolución, de ahí que se les llame tecnologías disruptivas, es decir, algo que cambia de forma radical la manera de hacer las cosas (Christensen, 1997). Estos avances están liderados por el surgimiento de la inteligencia artificial, la robótica, Internet de las cosas, vehículos autónomos, bio y nanotecnología, impresión tridimensional (3D), ciencia de los materiales, almacenamiento de energía (Diwan, 2017) y computación cuántica (Moret, 2013).

De acuerdo a la Liga de Universidades Europeas de Investigación, por sus siglas en inglés, LERU (2016), esta revolución, también conocida como la economía del conocimiento es la que está relacionada con la información, las comunicaciones, la educación y la innovación tecnológica; y el desarrollo en investigación, la robótica, y la nanotecnología entre otras disciplinas, de tal forma que, desde finales del siglo XX, la inversión en "capital intangible" (conocimiento, habilidades, actitudes) ha crecido considerablemente incluso en mayor medida que el capital tangible (maquinaria, materias primas), etc. 
Debido a lo anterior, en los EE. UU., el Partnershipfor 21st Century Learning [P21] (sf) ha desarrollado una visión muy popular dirigida al éxito de los estudiantes en la nueva economía global y ha identificado las habilidades, el conocimiento y la experiencia que se deben dominar para tener éxito en el trabajo y la vida en el mundo en el Siglo XXI. A continuación se describen estos elementos principales:

1. Preparación en temas clave y temas del siglo XXI: el ejemplo de temas clave incluiría: Inglés, Matemáticas, Ciencias y Artes.

2. Ejemplos de temas del siglo XXI serían: Conciencia Global, conocimiento práctico cívico y conocimiento práctico de la salud.

3. Habilidades de aprendizaje e innovación: creatividad e innovación; pensamiento crítico y solución de problemas; y comunicación y colaboración.

4. Información: habilidades en el uso de medios y tecnología digitales; conocimientos prácticos en información; conocimiento práctico de medios; y conocimientos prácticos en las TIC (Información, Comunicaciones y Tecnología).

5. Habilidades de vida y de la carrera: flexibilidad y adaptabilidad; iniciativa y autodirección; $\mathrm{y}$, habilidades sociales $\mathrm{e}$ interculturales; $\mathrm{y}$ productividad $\mathrm{y}$ responsabilidad.

\section{Objetivo de la investigación}

El propósito de este trabajo es identificar el grado de pertinencia del Programa de Ingeniero en Ciencia y Tecnología de Alimentos (PICTA).

Para Fisk (2017), la pertinencia de cualquier programa educativo en la Cuarta Revolución Industrial o Industria 4.0, promueve que los estudiantes aprendan no solo las habilidades y los conocimientos necesarios, sino también a identificar la fuente para aprender estas habilidades y conocimientos. Es decir, el aprendizaje se basa en ellos en cuanto a dónde y cómo aprender y el seguimiento de su rendimiento se realiza a través de la personalización basada en datos. Los compañeros se vuelven muy importantes en su aprendizaje. Aprenden juntos unos de otros, mientras que los maestros asumen el papel de facilitadores en su aprendizaje.

\section{Revisión de Literatura.}

En una investigación sobre este tema Hussin, (2018) reporta las diez habilidades que deben reunir los egresados frente a los retos que presenta la Cuarta Revolución Industrial, también conocida como Industria 4.0, siendo estas: Solución de Problemas Complejos, Pensamiento Crítico, Creatividad, Gestión de Personal, Coordinación con Otros, Inteligencia Emocional, Juicio y Toma de Decisiones, Orientación a los Servicios, Negociación y Flexibilidad Cognitiva, las cuales serán explicadas a continuación. 


\section{Solución de problemas complejos}

Wüstenberg (2011), señala que la resolución de problemas complejos (CPS por sus siglas en inglés) es la interacción exitosa con entornos de tareas que son dinámicas (es decir, cambian en función de la intervención del usuario y/o en función del tiempo) y en la que algunas, si no todas, las regularidades del entorno solo pueden ser reveladas por la exitosa exploración e integración de la información obtenida en ese proceso.

Las principales diferencias entre las tareas de razonamiento y CPS, son que en este último caso (1) no toda la información necesaria para resolver el problema se da desde el principio, (2) para el que resuelve el problema es necesario que genere activamente información a través de aplicar estrategias adecuadas, y (3) las habilidades de procedimiento tienen que usarse para controlar un sistema determinado, como cuando se utiliza la retroalimentación para persistir o cambiar el comportamiento o para contrarrestar los desarrollos no deseados iniciados por el sistema (Funke, 2001).

En resumen, a nivel conceptual, el razonamiento y la CPS evalúan las habilidades cognitivas necesarias para generar y aplicar reglas, las cuales deben generar correlaciones entre ambos constructos. Sin embargo, dependiendo de las diferentes características de la tarea y los procesos cognitivos descritos anteriormente, la CPS también debe mostrar validez divergente al razonamiento.

Por otra parte, Lohman y Michael (2000), en su investigación mencionan que la resolución de problemas es un aspecto importante de la práctica profesional. Los profesionales deben poder "dar un nuevo sentido a situaciones inciertas, únicas o conflictivas" que enfrentan regularmente en su trabajo (Schön, 1987, p. 39). Este tipo de situaciones, comúnmente conocidas como problemas mal estructurados, carecen de una identificación clara del problema, procedimientos para identificar soluciones y criterios para evaluar soluciones (Frederiksen, 1984). Cada vez más, los programas académicos están reconociendo la necesidad de que los profesionales puedan resolver problemas mal estructurados y están incorporando experiencias de instrucción en sus currícula para ayudar a los estudiantes a desarrollar la habilidad para resolver problemas (Mandin, Jones, Woloschuk, y Harasym, 1997).

\section{Creatividad}

En una investigación reciente, Puncreobutr (2016), menciona que los cambios en la economía social han ido evolucionando constantemente. Formalmente, fue la era de la Economía 1.0, hasta que se convirtió en la economía social digital, la era de la Economía 4.0, que juega un papel importante en el cambio de estilo de vida.

El autor hace énfasis en que "las habilidades innovadoras para vivir en la era de la Educación 4.0, además de poseer habilidades del siglo XXI que consisten en liderazgo, 
colaboración, creatividad, alfabetización digital, comunicación efectiva, inteligencia emocional, emprendimiento, ciudadano global, resolución de problemas y trabajo en equipo;también debe incluir las habilidades para construir una nación inteligente o personas inteligentes con pensamiento crítico, creatividad e innovación, comprensión intercultural, alfabetización informacional y mediática, habilidades profesionales y de aprendizaje" (Puncreobutr, 2016, p. 94).

La Educación 4.0 satisface las necesidades de la sociedad en una "era innovadora" (Puncreobutr, 2016, p.3). Está en concordancia con el comportamiento cambiante con las características especiales del paralelismo, conectivismo y visualización (Goldie, 2016). Esta gestión del aprendizaje debe ayudar a desarrollar en el estudiante, la capacidad para aplicar la nueva tecnología, que le ayudará a desarrollarse a las exigencias de los cambios en la sociedad.

De igual forma Terkowsky y Haertrel (2013) en su ensayo Fostering the Creative Attitude with Remote Lab Learning Environments, mencionan que la creatividad ha sido proclamada como una de las habilidades más importantes del siglo XXI.

Este ensayo ligeramente provocador induce posibles condiciones límite y restricciones para fomentar la creatividad en la educación en ingeniería. Además, presenta los primeros resultados de un pequeño estudio preliminar sobre los planes de estudio de educación superior de ingeniería, realizado en el proyecto alemán "ELLI - Enseñanza y Aprendizaje en la Educación en Ingeniería", lo que sugiere una falta de educación creativa en los currículos examinados. Además, presenta los resultados de un análisis descriptivo del enfoque didáctico del proyecto finalizado de la UE denominado "Pe-TEX - Plataforma para el Aprendizaje electrónico y la Experimentación Telemétrica" que proporciona información sobre las posibilidades de fomentar la actitud creativa en la educación de ingeniería mediante laboratorios remotos.

\section{Flexibilidad cognitiva}

La flexibilidad cognitiva se define como el ajuste a situaciones específicas, la capacidad de pasar de una idea a otra o la capacidad de considerar varios problemas en términos de múltiples dimensiones (Stevens, 2009), significa el hecho de que el individuo es consciente de sus opciones, puede adaptarse a nuevas situaciones y se siente competente para ellas (Bilgin, 2009).

Desde esta definición, Bülent (2013) desarrolló una investigación en la que se analizaron las contribuciones de apego, creencias irracionales y síntomas psicológicos a la predicción de la flexibilidad cognitiva. Se tomó una muestra de 436 alumnos que estudiaban en diferentes departamentos y facultades en la Universidad de Mersin. Se aplicaron las Escalas de Flexibilidad Cognitiva, la de Relaciones, la de Creencias Irracionales y el Inventario de Síntomas Cortos. Se utilizó el análisis de regresión múltiple y el análisis de regresión por pasos para analizar los datos. 
Los resultados de la investigación, demostraron que todas las variables independientes explicaban aproximadamente el $41 \%$ de la flexibilidad cognitiva; de acuerdo con el análisis de regresión por pasos, se descubrió que la flexibilidad cognitiva de los estudiantes se predecía más por las variables de creencias irracionales, apego obsesivo y ansiedad (Bülent, 2013).

De igual forma, Esen-Aygun (2018) llevó a cabo un trabajo de investigación cuyo propósito fue indagar la flexibilidad cognitiva de profesores practicantes en términos de variables específicas y determinar la relación entre la flexibilidad cognitiva de los profesores en formación y las habilidades de resolución de problemas interpersonales. El estudio se diseñó mediante el modelo de correlación descriptiva. Los datos se recolectaron a través del Inventario de Flexibilidad Cognitiva e Inventario de Solución de Problemas Interpersonales de 531 maestros que estudiaban en los Departamentos de Capacitación Primaria durante el semestre de otoño de los años académicos 2017-2018.

Los hallazgos indicaron que hubo diferencias significativas según el género y el nivel de la educación materna, mientras que no hubo diferencias significativas según el nivel de clase socioeconómica, el departamento académico y el nivel de educación de los padres, ni el estado socioeconómico y socio cultural. Además, los resultados demostraron que existía una relación entre la flexibilidad cognitiva de los maestros y las habilidades de resolución de problemas interpersonales.

\section{Pensamiento crítico}

De acuerdo a la American Philosophical Association (APA), el pensamiento crítico se conceptualiza para incluir tanto las habilidades cognitivas así como disposiciones afectivas (Facione, 1990).

Desde este concepto, Kong, Qin, Zhou, Mou y Gao (2014), desarrollaron una revisión sistemática de la literatura y un meta-análisis para estimar la efectividad del aprendizaje basado en problemas en el desarrollo del pensamiento crítico de estudiantes de enfermería.

Se incluyeron en el meta-análisis nueve artículos que representaban ocho ensayos controlados aleatorios. La mayoría de los estudios fueron de bajo riesgo de sesgo. El tamaño del efecto combinado mostró que el aprendizaje basado en problemas pudo mejorar el pensamiento crítico de los estudiantes de enfermería (puntajes generales de pensamiento crítico DME $=0,33$, IC 95\% $=0,13-0,52, p=0,0009$ ), en comparación con las clases tradicionales. Hubo baja heterogeneidad (puntajes generales de pensamiento crítico $\mathrm{I}^{2}=45 \%, \mathrm{p}=0.07$ ) en el meta-análisis. No se observó un sesgo de publicación significativo con respecto a las puntuaciones generales de pensamiento crítico ( $\mathrm{p}=$ 0,536). El análisis de sensibilidad mostró que el resultado del meta-análisis fue confiable.

La mayoría de los tamaños de efecto para las sub-escalas del Inventario de 
Disposiciones de Pensamiento Crítico de California (CCTDI) y la Taxonomía de Bloom favorecieron el aprendizaje problematizado, mientras que los tamaños de efecto para todas las sub-escalas de Habilidades de Pensamiento Crítico de la Prueba de California (CCTST) y la mayoría de las sub-escalas de la Evaluación del Pensamiento Crítico de Watson-Glaser (WCGTA) no fueron concluyentes.

Por lo tanto, los resultados del meta-análisis indicaron que el aprendizaje basado en problemas podría ayudar a los estudiantes de enfermería a mejorar su pensamiento crítico.

De igual forma Jeong (2003), examinó la interacción grupal y el pensamiento crítico en discusiones en línea. Para ello, utilizó la herramienta de análisis de discusión (DAT por sus siglas en inglés) para identificar patrones en las interacciones y determinar cuáles promovieron el pensamiento crítico. Con DAT, las transcripciones de la discusión se codificaron a través de doce eventos de pensamiento crítico, y las probabilidades de transición entre eventos se calcularon utilizando el método de análisis secuencial (Bakeman y Quera, 1995).

Al calcular las probabilidades de transición, DAT generó descripciones cuantitativas útiles de los patrones de interacción y los eventos de pensamiento crítico que le siguieron. Los hallazgos mostraron que las interacciones que involucran los puntos de vista en conflicto promovieron una mayor discusión y pensamiento crítico. El autor concluye que las herramientas como DAT serán útiles para probar empíricamente las interacciones y estructuras que mejoren las discusiones en línea.

\section{Inteligencia emocional}

De acuerdo al World Economic Forum Report (2016), una de las diez principales habilidades o competencias que deben desarrollar los estudiantes del siglo XXI para ingresar al mercado laboral de la Industria 4.0, es la inteligencia emocional.

La inteligencia emocional se ha definido como "un conjunto de habilidades que nos permite abrirnos camino en un mundo complejo: los aspectos personales, sociales y de supervivencia de inteligencia general, el sentido común y la sensibilidad evasivos que son esenciales para el funcionamiento diario efectivo" (Stein y Book, 2011, p. 14).

Jameson, Carthy, McGuiness \& McSweeney (2016) llevaron a cabo una investigación mediante la aplicación de una encuesta inicial de las opiniones de los empleadores ( $\mathrm{n}=$ 500) sobre la importancia y nivel actual de las competencias sociales y emocionales de estudiantes graduados en cinco sectores: IT / informática, servicios profesionales (incluidos contabilidad, negocios, finanzas, recursos humanos, derecho, comercio minorista), ciencias (incluidos farmacéutico y de la vida), y las ciencias sociales que se identifican en industrias en su etapa de crecimiento en Irlanda. 
Se buscó explorar las perspectivas de los empleadores para determinar si existen diferencias en cuanto a los requisitos de competencia social y emocional entre los graduados, en los diferentes sectores de empleo. Los hallazgos preliminares de la encuesta demostraron una gran disparidad entre el grado de importancia, atribuido por los empleadores a las competencias de inteligencia emocional y los niveles actuales mostrados por los empleados. Esto representa potencialmente una oportunidad significativa para permitir que los estudiantes desarrollen las habilidades específicas más favorecidas por los empleadores en sus áreas de carrera elegidas, lo que posiblemente incrementará su empleabilidad y éxito en el trabajo.

\section{Gestión de personal}

Recientemente, la atención en la investigación de la gestión de recursos humanos ha pasado de la estrategia al concepto de sistemas de "trabajo de alto rendimiento" (Colombo, Delamastro y Rabbiosi, 2007, p. 1038).

Recientemente, estudios empíricos realizados por académicos de gestión de recursos humanos han intentado mapear el desempeño de la innovación en relación con las prácticas de gestión de recursos humanos. El estudio de Jiménez-Jiménez y Sanz-Valle (2005) sobre una gama de empresas españolas examina cómo una empresa configura una estrategia de gestión de recursos humanos para el desempeño de la innovación. En este estudio de 350 firmas españolas, encontraron que el modelo de estrategia de Schuler y Jackson (1987) parece resultar en niveles más altos de desempeño de innovación entre las firmas de la muestra, que en las prácticas de gestión de recursos humanos.

Otro estudio empírico de firmas españolas realizado por Perdomo-Ortiz, GonzálezBenito y Galende (2009) examina el uso de prácticas de gestión de recursos humanos asociadas con la gestión de calidad total (TQM), denominadas "prácticas de gestión de recursos humanos TQM", y su impacto en el desempeño de la innovación de las empresas. Estos incluyen el trabajo en equipo, la capacitación extensa de los empleados, la gestión del desempeño y las medidas para aumentar la motivación de los empleados. En particular, los autores analizan el uso de estas prácticas en paquetes. Las prácticas de gestión de recursos humanos de TQM son muy similares a las prácticas asociadas con los sistemas de trabajo de alto rendimiento, por lo que el estudio presenta un proxy útil para el impacto de los sistemas de trabajo de alto rendimiento en el desempeño de la innovación. Los autores encuentran un vínculo directo entre el uso de paquetes en trabajos de alto rendimiento, prácticas de sistemas y desempeño de la innovación. Los vínculos más fuertes en el estudio son entre el uso del trabajo en equipo (organización del trabajo) y las medidas para aumentar la motivación de los trabajadores. También existe un vínculo directo más débil entre el uso de la capacitación y la innovación.

\section{Coordinación con otros}

En lo que se refiere a esta habilidad, Coskun, Kayikci \& Gencay (2019), propusieron un 
marco general de referencia para la educación superior enfocada a la Industria 4.0, que consistió en tres componentes principales: curriculum, laboratorio y club estudiantil para adaptar la formación en ingeniería a la visión de la Industria 4.0. En el componente del plan de estudios, determinaron la introducción de nuevos módulos de estudio y los cambios en los módulos de estudio existentes. En el siguiente paso, diseñaron dos laboratorios principales para abordar los cambios que hicieron en los planes de estudio, a saber, el Laboratorio de Producción Visual y el Laboratorio de Lego. Al definir la relación entre las horas del curso y las unidades prácticas de laboratorio, y cómo se llevarán a cabo estas unidades de laboratorio, utilizaron la Teoría del aprendizaje experiencial de Kolb (2014). En el último paso, mostraron cómo un club de estudiantes puede complementar los cambios definidos en los primeros dos pasos. Los estudiantes pueden tomar la iniciativa para proyectos relacionados con la Industria 4.0 bajo el techo de un club de estudiantes, que a su vez apoya la implementación de la etapa de experimentación activa de la Teoría del aprendizaje experiencial de Kolb. Los resultados preliminares de la implementación de este marco en la Universidad alemana de Turquía mostraron que era factible aplicar dicho marco y la teoría subyacente adoptada de Kolb para adaptar la educación en ingeniería a la visión de la Industria 4.0.

Así mismo, Hecklau, Galeitzke, Flachs y Kohl (2016), en su investigación mencionan que para hacer frente a los desafíos de conocimiento y competencia relacionados con las nuevas tecnologías y procesos de la Industria 4.0, se necesitan nuevos enfoques estratégicos para la gestión integral de recursos humanos en las empresas de manufactura. Debido a la automatización continua de los procesos de fabricación simples, el número de espacios de trabajo con un alto nivel de complejidad aumentará, lo que se traduce en la necesidad de un alto nivel de educación del personal. El reto es capacitar a los empleados para cambiar sus capacidades a espacios de actividades con procesos más complejos y garantizar la retención de puestos de trabajo en entornos de trabajo cambiantes. A pesar de que cada trabajo tiene diferentes requisitos, las competencias identificadas son cada vez más importantes y deben ser abordadas por el desarrollo de recursos humanos.

\section{Orientación al servicio}

Pérez-Barco (2017) menciona que a partir de ahora esta Cuarta Revolución Industrial será para trabajadores altamente cualificados y con una gran capacidad de adaptación, flexibilidad y aprendizaje continuo. Los que tengan menor formación serán desterrados. "El mercado tecnológico demanda perfiles más multidisciplinares, profesionales con un currículo en competencias en ciencias, tecnología, ingeniería y matemáticas (STEM por sus siglas en inglés), ya sea por educación universitaria o por un perfil más técnico procedente de formación profesional superior" (p.5).

El trabajador del futuro deberá poseer competencias como "el trabajo colaborativo y en equipo, la gestión del tiempo, resolución de problemas, razonamiento analítico, capacidad 
de buscar, filtrar y priorizar información" (p. 9).

De igual forma, la Fundación $1^{\circ}$ de Mayo (2016) en su informe hace énfasis en que 'Las habilidades hard deberán enseñarse en cada situación específica y girarán en torno a la comprensión de los sistemas, habilidades para cambios de herramientas en máquina, programación desde el nivel usuario hasta habilidades de programación más sofisticadas. Las habilidades soft cobran importancia máxima: flexibilidad, trabajo en equipo, capacidad de aprendizaje, etc." (p. 7). Desde el punto de vista de contenidos técnicos, es evidente "la necesidad de hibridación de habilidades TICs o entornos virtuales con mecánica, mecatrónica, ingeniería de sistemas, programación, diseño, etc. huyendo de la híperespecialización. Desde otro punto de vista, la ingeniería de organización debe complementarse con capacidades de gestión y organizativas" (Cataldi, Donnamaría y Lage 2009, p.82).

\section{Negociación}

De acuerdo a Rao (2017) la negociación es el proceso de dos personas o grupos que resuelven sus conflictos o problemas y logran una solución aceptable. El rol del negociador es generar confianza en las partes interesadas para resolver sus diferencias al llevarlas a un terreno común y lograr un resultado que beneficie a todos.

El autor menciona que en la era de la Industria 4.0, las habilidades de negociación son una parte integral del liderazgo suave, porque este liderazgo implica el uso de la persuasión y la negociación con la intención de lograr un resultado de ganar-ganar.

\section{Juicio y toma de decisiones}

Durante las últimas tres décadas, los investigadores interesados en las emociones y la cognición han intentado comprender la relación que el afecto y las emociones tienen con los resultados cognitivos, como juicio y toma de decisiones. Investigaciones recientes han revelado la importancia de examinar más las emociones discretas, que muestran que las emociones de la misma valencia (por ejemplo, la ira y el miedo) tienen un impacto diferencial como por ejemplo los resultados de juicio y decisión. Las revisiones narrativas de la literatura (Lerner \& Tiedens, 2006; Pham, 2007) han identificado algunos temas poco investigados, pero proporcionan una síntesis limitada de recomendaciones.

Debido a lo anterior, Angie, Conelly, Waples y Kligyte (2011), realizaron una investigación que examina la influencia de las emociones discretas en cuanto al resultado del juicio y la toma de decisiones y proporcionan una evaluación de los efectos observados utilizando un enfoque meta analítico. Los resultados, en general, mostraron que las emociones discretas tienen efectos de moderados a grandes en el juicio y en los resultados de la toma de decisiones. Sin embargo, los análisis de moderadores revelaron efectos diferenciales para las características de diseño del estudio y las características de manipulación de la emoción por tipo de emoción. 


\section{Pregunta de Investigación}

En relación con lo anterior, resulta inminente indagar en profundidad, formulando la siguiente pregunta:

¿Cuáles de las diez habilidades o competencias de las propuestas por Hussin (2018), son desarrolladas a partir del Programa Docente de Ingeniero en Ciencia y Tecnología de Alimentos (ICTA) para enfrentar los retos de la Cuarta Revolución Industrial o Industria 4.0?

\section{Método}

La metodología que se utilizó para esta investigación fue la aplicación de una encuesta como instrumento de medición, que utilizó una escala de tipo ordinal, o de tipo discreto y se basó en una escala de Likert de cinco puntos 1 (Completamente en Desacuerdo), 2 (En Desacuerdo), 3 (Neutra), 4 (De Acuerdo), y 5 (Completamente de Acuerdo), la cual recolectó los datos demográficos y las habilidades o competencias que el programa ayuda a desarrollar en los estudiantes durante su formación académica, la confirmación por parte de los egresados de Ingeniería en Ciencia y Tecnología de Alimentos en cuanto a la adquisición de estas habilidades o competencias, que demanda la Cuarta Revolución Industrial o Industria 4.0.

Las categorías que se investigaron fueron las diez habilidades o competencias que demanda el mercado laboral de la Cuarta Revolución Industrial o Industria 4.0, para los egresados de cualquier programa docente de nivel superior (Hussin, 2018).

Para medir la confiabilidad del instrumento debido a la naturaleza de sus variables continuas (totalmente de acuerdo a totalmente en desacuerdo), se utilizó el coeficiente de alfa Cronbach, que sirve para comprobar si el instrumento recopila información defectuosa y, por tanto, llevaría a conclusiones equivocadas o si se trata de un instrumento fiable que hace mediciones estables y consistentes (Creswell, 2008).

Para ello, se hizo una prueba piloto del instrumento, aplicándolo a seis profesores de adscritos a diferentes departamentos. El coeficiente de Alfa que se obtuvo fue de .85 , lo cual muestra que es un instrumento confiable (Morgan, Reichert \& Harrison 2002).

Una vez obtenida la confiabilidad del instrumento, se procedió a aplicarlo a una población de 60 egresados y a 12 profesores adscritos al programa. Es importante aclarar, que de los egresados del programa de ICTA, solamente respondieron la encuesta 14 egresados de dicho programa a diferencia de la población de los profesores, quienes en su totalidad la respondieron.

La razón por la que se seleccionó a profesores y egresados del PDICTA, fue con la 
finalidad de identificar si los profesores están actualizados en sus conocimientos de acuerdo lo que está exigiendo la Industria 4.0 y de ser así, hasta qué nivel los están impartiendo a los egresados en esta disciplina del conocimiento.

Los datos obtenidos de las encuestas tanto de egresados como de profesores del programa de ICTA, se analizaron mediante la técnica de estadística descriptiva empleando el programa Statistical Packagefor the Social Sciences 19.0 (SPSS, por sus siglas en inglés).

\section{Limitaciones}

Una de las limitaciones es la participación y respuesta por parte de los egresados y profesores para poder obtener una mayor cantidad de resultados, ya que no todos los invitados a participar respondieron positivamente.

La certeza de que los participantes en el estudio - los egresados del programa docente (ICTA) - tengan la claridad del significado de las habilidades o competencias que exige la Industria 4.0, en cuanto a su preparación y si perciben el significado de pertinencia.

\section{Resultados}

La encuesta se aplicó los 34 profesores de tiempo completo del PDICTA, y solamente la contestaron 12 profesores de los cuales el $75 \%$ fueron hombres y $25 \%$ mujeres, como se muestra en la tabla 1.

Tabla 1

Número de profesores del PD ICTA por género que dieron respuesta a la encuesta

\begin{tabular}{ccc}
\hline Género & $\boldsymbol{f}$ & $\%$ \\
\hline Hombres & 9 & 75 \\
Mujeres & 3 & 25 \\
Total & 12 & 100 \\
\hline
\end{tabular}

Fuente: Elaboración propia (2019).

En cuanto al grado académico de los profesores, como se muestra en la tabla 2 , el $8 \%$ tiene el grado de licenciatura, el $50 \%$ tiene el grado de maestría y el $42 \%$ posee el grado de doctor. 
Tabla 2

Grado académico de los profesores del PDICTA

\begin{tabular}{ccc}
\hline Grado académico & $\boldsymbol{f}$ & $\%$ \\
\hline Licenciatura & 1 & 8 \\
Maestría & 6 & 50 \\
Doctorado & 5 & 42 \\
Total & 12 & 100 \\
\hline
\end{tabular}

Fuente: Elaboración propia (2019).

Así mismo, la encuesta se aplicó a una población de 60 egresados que han mantenido comunicación con el jefe del PDICTA desde el año 2013; de esta población, solamente 14 egresados dieron respuesta a la encuesta de los cuales el $21 \%$ correspondieron al género femenino y $79 \%$ al género masculino, como se muestra en la tabla 3.

Tabla 3

Número de egresados de ICTA por género y porcentaje que dieron respuesta a la encuesta

\begin{tabular}{ccc}
\hline Género & $\boldsymbol{f}$ & $\%$ \\
\hline Hombres & 11 & 78.6 \\
Mujeres & 3 & 21.4 \\
Total & 14 & 100 \\
\hline
\end{tabular}

Fuente: Elaboración propia (2019)

Cabe mencionar que los egresados que participaron en dar respuesta a la encuesta, desde la fecha de su egreso, laboran en diferentes giros de empresas alimentarias, distribuidos de la siguiente manera: el 28\% trabaja en proceso y conservación de alimentos, el $14 \%$ en envases, el $7 \%$ en el ramo de materias primas para alimentos, mientras que el $14 \%$ labora en la tecnología de alimentos y el $35 \%$ lo hace en empresas de lácteos, como se muestra en la tabla 4.

Tabla 4

Distribución de egresados del PDICTA por giro de empresa

\begin{tabular}{ccc}
\hline & $\boldsymbol{f}$ & $\boldsymbol{\%}$ \\
\hline Proceso y conservación & 4 & 28.6 \\
Envases para alimentos & 2 & 14.3 \\
Materias primas para alimentos & 1 & 7.1 \\
Tecnología de alimentos & 2 & 14.3 \\
Lácteos & 5 & 35.7 \\
Total & 14 & 100 \\
\hline
\end{tabular}

Fuente: Elaboración propia (2019).

Para dar respuesta a la pregunta de investigación se usó el análisis estadístico descriptivo utilizando el paquete estadístico SPSS 19.0. Los datos obtenidos de este análisis, para cada una de las diez habilidades, de la encuesta aplicada a los profesores y egresados del PDICTA, se muestran en la tabla 5 con su explicación respectiva. 


\section{Análisis de resultados}

En la tabla 5 se muestran los resultados obtenidos de la estadística descriptiva donde los valores de las variables que tienen mayor frecuencia son los que se refieren a las habilidades o competencias de solución de problemas complejos, creatividad, juicio crítico y toma de decisiones, y flexibilidad cognitiva. En otras palabras, el valor de la moda (Mo= 4) evidenció que tanto los profesores como los egresados, estuvieron de acuerdo en que los profesores apoyaron a los egresados en el desarrollo de las habilidades.

Para las variables de pensamiento crítico, gestión de personal, coordinación con otros, orientación a los servicios, negociación e inteligencia emocional, entre otros, los resultados se expresaron de acuerdo a la escala de calificación proporcionada 1(estar totalmente en desacuerdo), 2 (estar en desacuerdo), 3 (neutral), 4 (estar de acuerdo) y representada con los niveles correspondientes (tabla 5).

De acuerdo con la moda representada con un nivel o calificación de 4 para las variables de solución de problemas complejos, creatividad, juicio crítico y toma de decisiones, flexibilidad cognitiva, orientación a los servicios y negociación, los profesores estuvieron de acuerdo en haber apoyado en el desarrollo de estas habilidades en el egresado (tabla 5).

Analizando la moda obtenida del análisis descriptivo de los resultados de los egresados (tabla 5), ellos establecen con una moda máxima de 4 que los profesores participaron en el desarrollo de las habilidades de las variables de solución de problemas complejos, creatividad, juicio crítico y toma de decisiones, flexibilidad cognitiva, pensamiento crítico, e inteligencia emocional.

Los profesores expresaron con una moda de 2 (tabla 5) que no consideraron el desarrollo de habilidades de gestión de personal ni de inteligencia emocional, sin embargo, para el caso de los egresados realmente ellos afirmaron que en el caso de la inteligencia emocional sí se le desarrolló en esa habilidad.

Para las variables de gestión de personal, coordinación con otros, orientación a los servicios y negociación, los egresados evaluaron el desarrollo de las habilidades con una moda de 3 (neutral), lo cual nos indica que no consideran que se les desarrollaron las habilidades (tabla 5).

\section{Tabla 5}

Valores de la moda obtenidos de la estadística descriptiva como resultado de la encuesta aplicada a los profesores y egresados del PDICTA

\begin{tabular}{ccc}
\hline Habilidades o competencias & $\begin{array}{c}\left(\boldsymbol{M}_{\boldsymbol{o}}\right) \\
\text { profesores }\end{array}$ & $\begin{array}{c}\left(\mathbf{M}_{\boldsymbol{o}}\right) \\
\text { egresados }\end{array}$ \\
\hline Solución de problemas complejos & 4 & 4 \\
Creatividad & 4 & 4 \\
Juicio crítico y toma de decisiones & 4 & 4 \\
Flexibilidad cognitiva & 4 & 4 \\
Pensamiento crítico & 3 & 4
\end{tabular}




\begin{tabular}{ccc} 
Gestión de personal & 2 & 3 \\
Coordinación con otros & 3 & 3 \\
Orientación a los servicios & 4 & 3 \\
Negociación & 4 & 3 \\
Inteligencia emocional & 2 & 4 \\
\hline
\end{tabular}

Fuente: Elaboración propia (2019).

\section{Conclusiones}

A partir de los resultados de la investigación se presentan las siguientes conclusiones teniendo en cuenta la pregunta de investigación:

1. Partiendo de los resultados del análisis estadístico descriptivo, el PDICTA solamente cubre cuatro de las habilidades mencionadas (solución de problemas complejos, creatividad, juicio crítico y toma de decisiones, y flexibilidad).

2. De acuerdo al análisis se sustenta que, para adquirir las competencias o habilidades para la solución de problemas complejos, creatividad, juicio crítico, toma de decisiones y flexibilidad los egresados recibieron apoyo por parte de los profesores.

3. Considerando lo que menciona Hussin (2018) respecto a que los egresados de educación superior deben reunir las 10 habilidades o competencias que la Industria 4.0 demanda incorporarse al mercado laboral se concluye que el PDICTA tiene un avance del $40 \%$ en términos relativos.

\section{Recomendaciones}

Los resultados de este estudio permiten visualizar la situación actual de los de los profesores y egresados del PDICTA, en cuanto a las habilidades o competencias que demanda la Cuarta Revolución Industrial o Industria 4.0.

En relación con el resultado obtenido de las demás habilidades (pensamiento crítico, gestión de personal, coordinación con otros, inteligencia emocional, orientación al servicio y negociación), resulta evidente que la universidad debe considerar la actualización curricular del PDICTA, para que este pueda enfrentar las exigencias de la Industria 4.0.

Es necesario hacer investigaciones adicionales para determinar el grado en que los hallazgos de este estudio puedan generalizarse a los demás programas académicos y con ello identificar si tienen el mismo nivel de cobertura de habilidades o competencias que el PDICTA para enfrentar los retos de la Cuarta Revolución Industrial o Industria 4.0.

Es recomendable hacer una investigación con la población de los estudiantes del PDICTA, para verificar si ya se cubre la totalidad de las diez habilidades o competencias, 0 si para lograrlo, es necesario capacitar a los profesores y adquieran las competencias para 
ello.

Debido a la heterogeneidad de los programas educativos de la universidad, los resultados no se podrían generalizar y su aplicación sería exclusivamente aplicable al programa identificado.

La universidad debería enfocarse a la actualización curricular de este programa para estar preparada para enfrentar los retos de la Industria 4.0.

Finalmente, se ve la necesidad de sensibilizar a los docentes sobre el cambio que está surgiendo en el mercado laboral a nivel global, en la formación de talento humano para hacer frente a los retos que se mencionan en el párrafo anterior.

\section{Referencias}

Angie, A. Conelly, S., Waples, E. \& Kligyte, V. (2011). The influence of discrete emotions on judgement and decision-making: A metanalytic review. Cognition and Emotion 25(8), 1393-1422. DOI: http://dx.doi.org/10.1080/02699931.2010.550751

Bakeman, R. \& Quera, V. (1995). Analysing interaction: Sequential analysis with SDIS and GSEQ. Cambridge: Cambridge University Press.

Beugelsdijk, S. (2008). Strategy human resource practices and product innovation. Organization Studies, 29(6), 821-847.

Bilgin. M. (2009). Developing a cognitive flexibility scale: Validity and reliability studies. Social Behavior and Personality, 373), 343-354.

Bülent, G. (2013). The contributions to attachment styles, irrational beliefs and psychological symptoms to the prediction of cognitive flexibility. Educational Sciences: Theory and Practice, 13(4), 2079 - $2075 . \quad$ DOI: http://dx.doi.org/10.12738/estp.2013.4.170

Cataldi, Z., Donnamaría, M. C., \& Lage, F. (2009). Didáctica de la química y las TIC's: Laboratorios virtuales, modelos y simulaciones como agentes de motivación y de cambio conceptual. Recuperado de: http://hdl.handle.net/10915/18979

Colombo, M., Delmastro, M., \& Rabbiosi, L. (2007). 'High performance work practices, decentralisation, and profitability: evidence from panel data'. Industrial and Corporate Change, 16(6), 1037-67.

Coskun, S., Kayikci, Y., \& Gençay, E. (2019). Adapting engineering education to industry 4. vision. Technologies, 710$)$ 1-13. DOI: http://dx.doi.org/10.3390/technologies7010010 
Creswell, W. J. (2008). Educational research: Planning, conducting and evaluating quantitative and qualitative research. (3rd. ed). Upper Saddle River, NJ: Pearson Publishing.

Christensen, C. (1997). The innovator's dilemma: When new technologies cause great firms to fail.Harvard, Harvard University Press.

Diwan, P. (2017). Is Education 4.0 an imperative for success of 4thIndustrial Revolution? Recuperado de: https://medium.com/@pdiwan/is-education-4-0-an-imperative-forsuccess-of-4th-industrial-revolution-50c31451e8a4

Pappas, C. (June 2016). 5 Ways to promote negotiation skills through corporate learning. Recuperado de: https://elearningindustry.com/5-ways-promote-negotiation-skillscorporate-elearning

Esen-Aygun, H. (2018). The relationship between pre-service teachers' cognitive flexibility and interpersonal problem solving skills. Eurasian Journal of Educational Research77, (2018), 105-128.

Facione, P. (1990). The delphi report. A statement of expert consensus for purposes of educational assessment and instruction. Millbrae, CA: The California Academic Press.

Fisk, P. (2017). Education 4.0 ... the future of learning will be dramatically different, in school and throughout life. Recuperado de: http://www.thegeniusworks.com/2017/01/future-education-young-everyone-taught-together

Frederiksen, N. (1984). Implications of cognitive theory for instruction in problem solving. Review of Educational Research 54(3), 363-407.

Fundación 1 de Mayo (2016, Junio). Aproximación a las cualificaciones profesionales en la Industria 4.0. Recuperado de: http://www.relats.org/documentos/ET.AcostaPerez2.pdf

Funke, J. (2001). Dynamic systems as tools for analysing human judgement. Thinking and Reasoning (7), 69-89.

Goldie, J. (2016). Connectivism: A knowledge learning theory for the digital age? Medical teacher, 38(10), 1064-1069. DOI: http://dx.doi.org/10.3109/0142159X.2016.1173661

Hecklau, F., Galeitzke, M., Flachs, S. \&Kohl, H. (2016). Holistic approach for human resource management in industry 4.0. Procedia CIRP 54(2016), 1-6. DOI: http://dx.doi.org/10.1016/j.procir.2016.05.102 
Hussin, A. A. (2018). Education 4.0 made simple: ideas for teaching. International Journal $\begin{array}{llll}\text { of Education } \& \text { Literacy } \quad \text { Studies, } & \text { 92-98. }\end{array}$ http://dx.doi.org/10.7575/aiac.ijels.v.6n.3p.92

Hermann, M., Pentek, T., \& Otto, B. (2015). Design principles for Industrie 4.0 scenarios: a literature review. Technische Universität Dortmund: Dortmund. Recuperado de: https://www.researchgate.net/publication/307864150_Design_Principles_for_Indus trie_40_Scenarios_A_Literature_Review

Jameson, A., Carthy, A., McGuiness, C., \& McSweeney, F. (2016). Emotional intelligence and graduates - employers' perspectives. Procedia - Social and Behavioral Sciences, 228(2016), 551-522. DOI: http://dx.doi.org/10.1016/j.sbspro.2016.07.079

Jeong, C. A. (2003). The sequential analysis of group interaction and critical thinking in online threaded discussions. The American Journal of Distance Education1入1), 25 $-43$.

Jimenez-Jimenez, D. \& Sanz-Valle, R. (2005). Innovation and human resource management fit: an empirical study. International Journal of Man Power 26(4). 364-381.

Kolb, D. (2014). Experiential learning as the science of learning and development. Englewood Cliffs, N.J: Prentice Hall.

Kong, L., Qin, B., Zhou, Y., Mou, S., \& Gao, H. (2014). The effectiveness of problem-based learning on development of nursing students' critical thinking: A systematic review and meta-analysis. International Journal of Nursing Studies, 51(2014), 458-469. DOI: http://dx.doi.org/10.1016/j.ijnurstu.2013.06.009

Lerner, J., \& Tiedens, L. (2006). Portrait of theangry decision maker: How appraisal tendenciesshape anger's influence on cognition. Journal of Behavioral DecisionMaking, 19(3), 115-137.

LERU. (2016).Asociación Multisectorial de Empresas de Tecnologías de la Información, Comunicaciones y Electrónica. Recuperado de: https://www.leru.org/publications

Lohman, M. C. (2000). Designin groups in problem-based learning to promote problem solving skill and self-directedness. Instructional Science 28(3), 291-307.

Mandin, H., Jones, A., Woloschuk, W. \& Harasym, P. (1997). Helping students learn to thinklike experts when solving clinical problems. Academic Medicine 72(3). 173179.

Moret, B. (2013). Principios fundamentales de computación cuántica. Recuperado de: 
htpp://www.lidiagroup.org/images/descargas/varios/011cuantica.pdf

Morgan, S. E., Reichert, T. \& Harrison, T. R. (2002). From numbers to words: Reporting statistical results for the social sciences. Allyn \& Bacon, Boston, MA.

Partnership for the 21st Century Learning [P21] (s. f.). framework for $21^{\text {st }}$ century learning. Recuperado de: http://tinyurl.com/o6urqgh

Perdomo-Ortiz, J., González-Benito, J. \& Galende, J. (2009). An analysis of the relationship between total quality management-based human resource management practices and innovation. The International Journal of Human Resource Management 20(5). 1191-2018.

Pérez-Barco, M. (2017). Las nuevas profesiones y habilidades que demanda la revolución tecnológica. Recuperado de: https://www.abc.es/economia/abci-nuevasprofesiones-y-habilidades-demanda-revolucion-tecnologica201603282128_noticia.html

Pham, M. (2007). Emotion and rationality: A critical review and interpretation of empirical evidence. Review of General Psychology, 11(2), 155-178.

Puncreobutr, V. (2016). Education 4.0: New challenge of learning. St. Theresa Journal of $\begin{array}{llll}\text { Humanitiesand Social } & \text { S(2). }\end{array}$ http://www.stic.ac.th/ojs/index.php/sjhs/article/view/Position\%20Paper3

Rao, M. S. (2017). Soft leadership training for improved negotiation skills. Recovered from: https://trainingindustry.com/blog/leadership/soft-leadership-training-forimproved-negotiation-skills/

Schön, D. (1987). Educating the reflective practitioner. San Francisco: Jossey-Bass.

Schuler, R.S. \& Jackson, S.E. (1987). Linking competitive strategies with human resource management practices. The Academy of Management Executive 1(3). 207-219.

Shwab, K. (2016). The Fourth Industrial Revolution: what it means, how to respond. Recovered from: https://www.weforum.org/agenda/2016/01/the-fourth industrialrevolution-what-it-means-and-how-to-respond

Stein, S. \& Book, H. (2011). The EQ edge: Emotional intelligence and your success. (3trd ed.). US: Multy-Health Systems Inc.

Stevens, A. (2009). Social problem-solving and cognitive flexibility: Relations to social skills and problem behavior of at-risk young children (Doctoral thesis). Recovered from: https://www.bookdepository.com/es/Social-Problem-Solving-Cognitive-FlexibilityRelations-Social-Skills-Problem-Behavior-At-Risk-Young-Children-Arianne-D- 


\section{Stevens/9781244025844}

Terkowsky, C., \& Haertel, T. (2013). Fostering the creative attitude with remote lab learning environments. International Journal of Online and Biomedical Egineering g(5), 13-20. http://dx.doi.org/10.3991/ijoe.v9iS5.2750

World Economic Forum Report (2016). Chapter 1: The future of jobs and skills. Recovered from: http://reports.weforum.org/future-of-jobs-2016/chapter-1-the-future-of-jobsand-skills

Wüstenberg, S., Greiff, S. \& Funke, J. (2011). Complex problem solving - more than reasoning? Intelligence 40(2012), 1-14. DOI: http://dx.doi.org/10.1016/j.intell.2011.11.003 